\title{
Solution processing route to Na incorporation in CZTSSe nanoparticle ink solar cells on foil substrate
}

\author{
Xinya $\mathrm{Xu}^{1}$ - Yongtao $\mathrm{Qu}^{1}$ - Stephen Campbell ${ }^{1}$ Mathieu Le Garrec ${ }^{1} \cdot$ Bethan Ford $^{1}$ - Vincent Barrioz ${ }^{1}$. \\ Guillaume Zoppi ${ }^{1} \cdot$ Neil S. Beattie ${ }^{1}$
}

Received: 20 November 2018 / Accepted: 9 March 2019 / Published online: 20 March 2019

(C) The Author(s) 2019

\begin{abstract}
Sodium is a key dopant in thin film photovoltaic cells with reported benefits including promotion of grain growth, passivation of grain boundaries and increased carrier concentration in chalcopyrite and kesterite based solar cells. Research-grade devices fabricated in substrate configuration often rely on diffusion of $\mathrm{Na}$ from a soda lime glass substrate into the photovoltaic absorber layer during high temperature processing. However, for samples on flexible substrates such as foils and plastics, this is not available and requires alternative approaches. In this work, we fabricate Earth-abundant $\mathrm{Cu}_{2} \mathrm{ZnSn}(\mathrm{S}, \mathrm{Se})_{4}$ thin film solar cells from nanoparticle inks on flexible molybdenum substrates and demonstrate a simple, low-cost route to incorporating $\mathrm{Na}$ in solution thereby making it compatible with large area, high volume manufacturing. The technique is verified to improve the device efficiency relative to a reference flexible device built on molybdenum foil.
\end{abstract}

\section{Introduction}

Flexible solar cells have the potential to enable new applications in small-to-medium scale distributed energy and must therefore be cost competitive and sustainable. Considerable success has been achieved with thin film $\mathrm{CuInGa}(\mathrm{S}, \mathrm{Se})_{2}$ (CIGS) on a polyimide (PI) substrate demonstrating up to $20.4 \%$ solar energy conversion efficiency [1]. Compared with CIGS, Earth abundant $\mathrm{Cu}_{2} \mathrm{ZnSn}(\mathrm{S}, \mathrm{Se})_{4}$ (CZTSSe) lags in performance and nowhere is this more evident than in the flexible domain with a record efficiency of $5.6 \%$ on a ferritic stainless steel foil substrate [2]. There is therefore a pressing need for fundamental research to address this challenge.

Irrespective of substrate choice, the incorporation of alkali dopants in both CIGS and CZTSSe is critical to achieve high performance and this is associated with a reduction in the density of grain boundaries which limit the collection of photo-generated carriers due to non-radiative recombination. For example, the formation of liquid $\mathrm{Na}_{2} \mathrm{Se}_{x}$ phases during annealing has been associated with improved grain growth [3] and favourable formation of (112) crystal

Neil S. Beattie

neil.beattie@northumbria.ac.uk

1 Department of Mathematics, Physics and Electrical Engineering, Northumbria University, Ellison Building, Newcastle upon Tyne NE1 8ST, UK facets $[4,5]$, as well as increasing the device open circuit voltage $\left(V_{O C}\right)$ and fill factor (FF) [6-9]. The improved $V_{O C}$ is explained as a higher built-in voltage resulting from movement of the bulk Fermi level toward the valence band thereby reducing the influence of deep recombination centres, while higher FF stems from increased bulk CZTSe conductivity with increased hole density and mobility [7]. For researchgrade thin film solar cells, the source of Na-doping has commonly been provided by a soda lime glass (SLG) substrate with diffusion of $\mathrm{Na}$ to the photovoltaic light absorbing layer occurring during a high temperature processing step [10]. Alternatively, for Na free substrates, sodium may be incorporated by doping the back contact $(\mathrm{MoNa})[6,11,12]$ or by introducing additional layers before or after the photovoltaic absorber formation [2, 13, 14].

Of all the fabrication routes to the production of CZTSSe solar cells, solution processing offers the highest efficiency [15]. Unfortunately, this process involves hydrazine which is highly toxic and relatively unstable. A less hazardous approach with a lower environmental impact involves the fabrication of $\mathrm{Cu}_{2} \mathrm{ZnSnS}_{4}$ nanoparticle inks and subsequent conversion to CZTSSe absorbers via a thermal annealing step. The highest reported efficiency for this fabrication technique is $9.3 \%$ [16]. Another attractive solution processing fabrication route to CZTS involves a solution of molecular precursors in which dissolving alkali dopants has been recently studied [17-19]. Both the nanoparticle ink 
and precursor approaches are compatible with a variety of scalable deposition techniques such as doctor blade printing $[20,21]$ and in this work we present a simple, low-cost $\mathrm{Na}$-doping approach using the nanoparticle ink approach to achieve a power conversion efficiency of $4.4 \%$ from CZTSSe solar cells built on flexible Mo-foil substrates.

\section{Experimental details}

Mo foil (99.9\%, Sigma Aldrich) was chosen as a flexible substrate due to its excellent thermal stability and high electrical conductivity. A Mo film $(\sim 1800 \mathrm{~nm})$ was sputtered directly onto the foils by direct current magnetron sputtering with target power density of $9 \mathrm{~mW} \mathrm{~cm}^{-2}$ and an Ar pressure of 7 mTorr at room temperature to reduce series resistance [22].

CZTS nanoparticle inks were chemically synthesised using the well-established hot injection of metallic precursors into surfactant and described in detail elsewhere [23]. $\mathrm{NaF}$ powder was dissolved in the obtained inks as a novel and effective doping method. The inks were deposited directly on the Mo-coated Mo foil substrate by spin coating to create a uniform thin film of densely packed CZTS nanoparticles. Repeated application of the spin coating process allowed the thickness of the resulting thin film to be controlled and after achieving the appropriate thickness $(1 \mu \mathrm{m})$, the films were heated in a selenium-rich atmosphere for $20 \mathrm{~min}$ at $500{ }^{\circ} \mathrm{C}$ to form a CZTSSe thin film photovoltaic absorbers.

For Na-doping, $1.9 \mathrm{mg}$ of $\mathrm{NaF}$ was added directly to a $1.5 \mathrm{~mL}$ of hexanethiol solution of CZTS nanoparticle inks with concentration $100 \mathrm{mg} / \mathrm{mL}$. NaF is chosen because of the high solubility and low cost. To evaluate the effect of introducing $\mathrm{Na}$ doping, reference devices without $\mathrm{NaF}$ were also fabricated on Mo foil substrates. With the exception of the introduction of $\mathrm{Na}$, the fabrication and processing of devices were nominally identical.

Solar cell devices were completed using a conventional thin film substrate approach with structure of Mo foil + sputtered Mo film substrate/CZTSSe/CdS /ZnO/ITO/Ni+Al contact grid. Further details of photovoltaic cell fabrication may be found in our previous publications [22, 23]. The $\mathrm{CdS}$ buffer layer was fabricated using chemical bath deposition with resulting thickness of $90 \mathrm{~nm}$. The $\mathrm{ZnO}$ and indium tin oxide (ITO) layers were magnetron sputtered before a $\mathrm{Ni} / \mathrm{Al}$ front contact grid was deposited by electron beam evaporation through a shadow mask. The area of the grid was $1.15 \mathrm{~mm}^{2}$. To ensure reproducibility of the photovoltaic performance data, a minimum of three devices were built for each doping condition.

The surface morphology of the CZTSSe absorber and its cross-sectional structures were studied by scanning electron microscopy (SEM, Tescan Mira 3) and elemental composition was determined by energy dispersive spectroscopy (EDS) attached to the SEM. The crystal structure was determined using X-ray diffraction (XRD, Siemens D-5000) with a $\operatorname{CuK}_{\alpha}$ radiation source $\left(\lambda=0.154 \mathrm{~nm}\right.$ for $\left.\mathrm{K}_{\alpha 1}\right)$. Secondary ion mass spectroscopy (SIMS) using a Hiden Analytical gas ion gun and quadrupole detector was used to obtain elemental depth profiles. Photocurrent density-voltage (J-V) characteristics of the CZTSSe solar cells were measured under standard air mass 1.5 solar illumination with an intensity of $100 \mathrm{~mW} \mathrm{~cm}^{-2}$ (Abet Technologies Sun 2000 solar simulator). External quantum efficiency (EQE) measurements were performed using a double grating monochromator with illumination normalised against calibrated $\mathrm{Si}$ and Ge detectors. Capacitance-voltage (C-V) profiles of solar cells were measured by an Agilent E4980A Precision LCR Meter assisted with Versa Studio sweeping with both forward and reverse AC biased voltage, and the maximum current limited to 10 $\mathrm{mA}$ with compliance.

\section{Results and discussion}

\subsection{Photovoltaic performance}

Figure 1a shows $J V$ characteristics for solar cells fabricated from doped and reference CZTSSe absorbers described above, under illuminated and dark conditions. Additionally, the extracted values of $V_{O C}$, short circuit current density $\left(J_{S C}\right)$, fill factor $(F F)$, efficiency $(\eta)$, series $\left(R_{s}\right)$ and shunt $\left(R_{s h}\right)$ resistance are given in Table 1 . The average and deviation values are shown in the brackets. It can be seen that the Na doped device on a flexible Mo foil substrate exhibits an efficiency of $4.4 \%$ which is a substantial improvement relative to the reference device. Table 1 indicates that the origin of this improvement is found in both $V_{O C}$ and $J_{S C}$. Notably both doped and reference flexible solar cells exhibit degraded $R_{s h}$ relative to a device on a rigid SLG substrate (data shown in ref [22]). However, it is interesting to note that within the limitations of the fabrication process the overall device efficiency for a
Table 1 Device performance for doped and reference samples. Average values and standard deviations are given in brackets

\begin{tabular}{lllllll}
\hline & $V_{O C}(\mathrm{mV})$ & $J_{S C}\left(\mathrm{~mA} \mathrm{~cm}^{-2}\right)$ & $\mathrm{FF}(\%)$ & $\eta(\%)$ & $R_{s h}\left(\Omega \mathrm{cm}^{2}\right)$ & $R_{s}\left(\Omega \mathrm{cm}^{2}\right)$ \\
\hline Doped & $300(300 \pm 7)$ & $33.9(33.7 \pm 0.4)$ & $43.5(41.9 \pm 1.3)$ & $4.4(4.2 \pm 0.2)$ & 39.1 & 3.4 \\
Reference & $250(240 \pm 8)$ & $25.3(24.0 \pm 1.3)$ & $39.3(38.5 \pm 0.9)$ & $2.5(2.4 \pm 0.1)$ & 33.4 & 4.5 \\
\hline
\end{tabular}




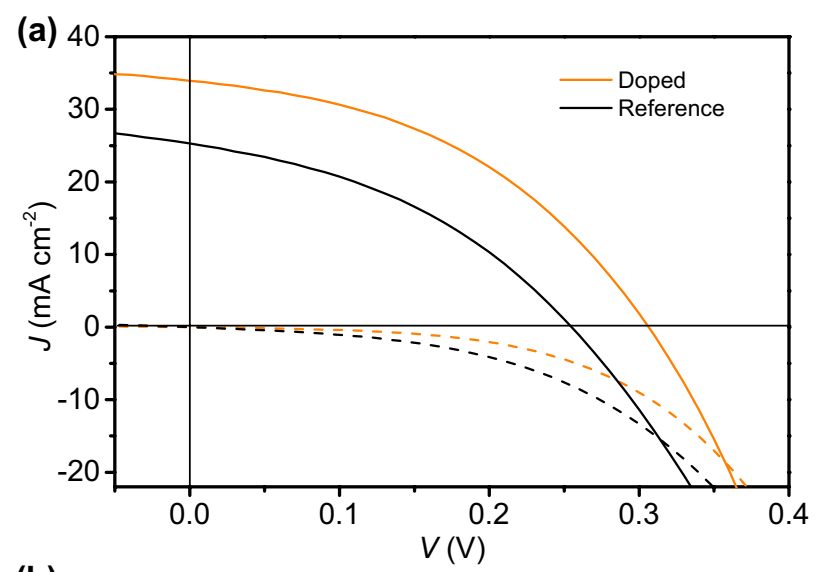

(b)

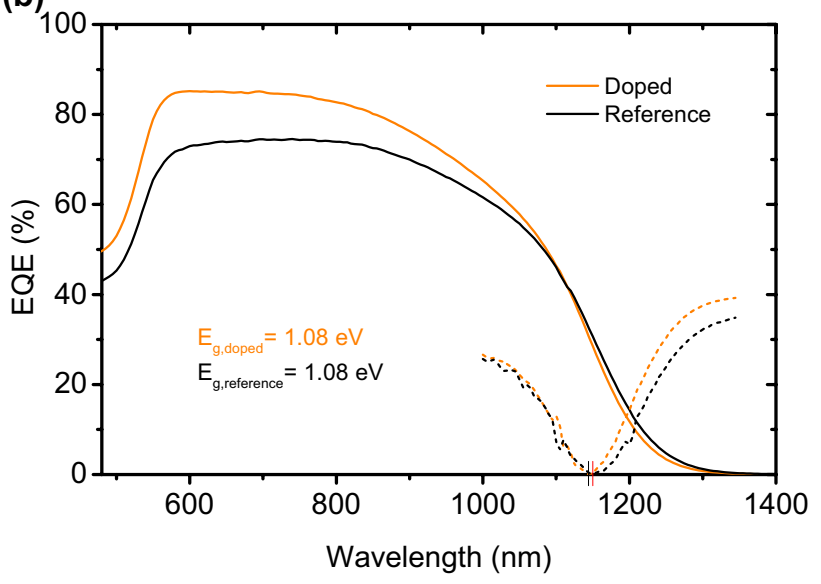

Fig. 1 a JV curves of cells prepared from doped and reference inks on Mo foil. b EQE spectra for two samples. The bandgap is estimated by the minimum value of the derivative of the EQE curves within the long-wavelength range

Na-doped CZTSSe solar cell on a Mo-foil substrate either matches or exceeds the performance of a nominally identical device built on SLG.

The EQE response for the devices are shown in Fig. 1b. The bandgap energy can be determined by the minimum value of the derivative in the long-wavelength region. The extracted bandgaps are both around $1.08 \mathrm{eV}$ indicating no significant influence of the $\mathrm{Na}$ on this fundamental optoelectronic property. Photocurrent $\left(J_{S C}\right)$ values were obtained by integration of $\mathrm{EQE}$ according to:

$J_{S C}=\int F(\lambda) \cdot \lambda \cdot E Q E(\lambda) \cdot q / h c d \lambda$,

where $F(\lambda)$ is the standard air mass (AM) 1.5 solar spectrum (International standard ISO 9845-1, 1992) [26], $\lambda$ is wavelength, $h$ is Planck's constant, and $c$ is speed of light. This yielded $30.2 \mathrm{~mA} \mathrm{~cm}^{-2}$ and $26.2 \mathrm{~mA} \mathrm{~cm}^{-2}$ (total area) for the doped and reference devices, respectively. These values are similar to those in Table 1 which were obtained from the
$J V$ measurement. In general, the EQE for the doped sample exhibits an overall improvement from short to long wavelengths. This indicates longer carrier diffusion length and reduced recombination in the doped sample. These effects have previously been associated with an increased absorption in the CTZSe absorber grain size [24, 25].

\subsection{Morphologies and composition}

In order to investigate the differences in AM 1.5 JV characteristics and EQE signals between the doped and reference flexible solar cells, SEM top view and cross-section images were obtained from selenised CZTSSe thin film absorbers as shown in Fig. 2. Top view images for the doped (Fig. 2a) and reference (Fig. 2b) qualitatively indicate that Na-doping yields smoother, homogeneous large grains resulting in a more uniform photovoltaic absorber. This improvement in grain properties may be correlated with the substantial increase in $J_{S C}$ observed in Table 1 between the devices. Cross-sectional images reveal less significant features however, it is notable that the characteristic fine grain layer [27] commonly observed between the Mo substrate and the CZTSSe absorber is much thinner $(20 \mathrm{~nm})$ in the doped sample compared with the reference sample $(350 \mathrm{~nm})$. In addition the Na-doped absorber is thinner than the reference case which is interesting because the device EQE (Fig. 1) is actually greater across the spectrum for Na-doped device suggesting better capacity for charge extraction than in the reference absorber. This may follow directly from the $\mathrm{Na}-$ doping technique because the dopants are assumed to be distributed evenly throughout the nanoparticle precursor layers and therefore the origin of Na required to promote grain growth is immediately available during the selenisation process. Thickness variations in the CZTSSe absorber layer between the samples were attributed to processing conditions and the point at which the cross-sectional SEM image was obtained as sample cleavage for imaging can result in discontinuities and strained layers.

The compositions of the thin film photovoltaic absorbers were determined from EDS line scans as shown in Fig. 3. Using these data, the elemental compositions of the CZTSSe photovoltaic absorbers were determined at $x=0.5 \mu \mathrm{m}$ (indicated by the dashed red lines in Fig. 3) and the values are given in Table 2. From these data it can be seen that both samples are highly selenised and $\mathrm{Cu}$ poor which is favourable for solar cell performance. Furthermore, in addition to being thinner, the fine grain layer in the doped sample is found to contain less $\mathrm{C}$ which is associated with residual ligand from the fabrication process.

XRD data for the doped and reference flexible solar cells are shown in Fig. 4a. The two peaks marked as orange stars are the reflection from $\mathrm{MoSe}_{x}$. The peak at $52.55^{\circ}$ marked as a grey star is a reflection from the strong peak at $58.67^{\circ}$ 
(a)
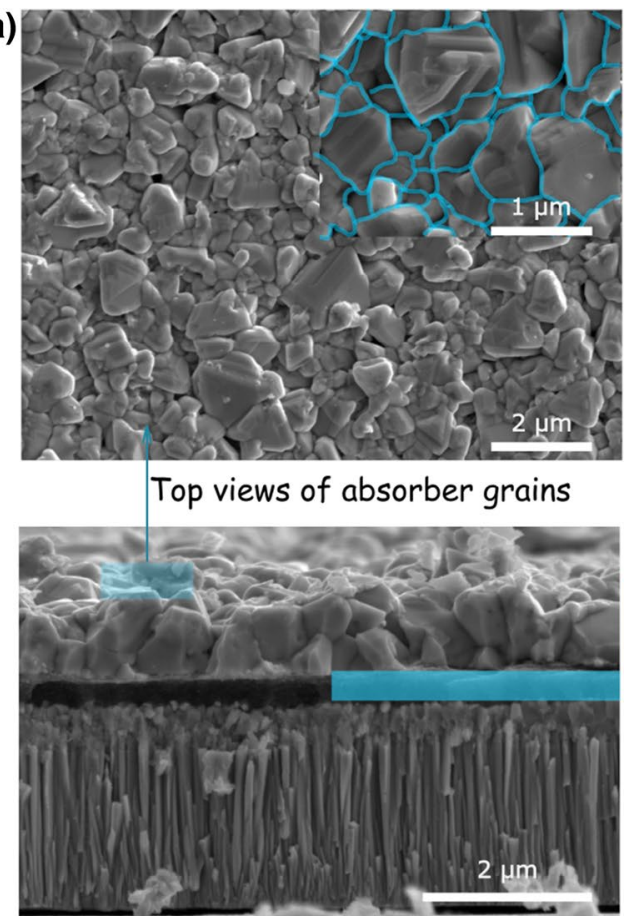

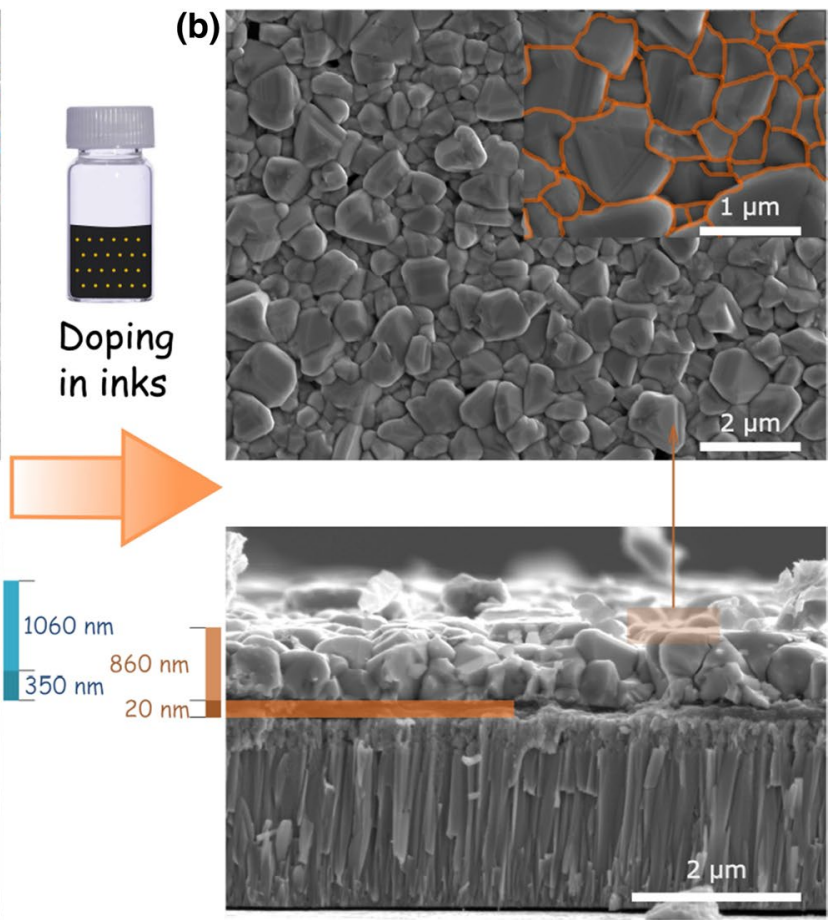

Fig. 2 Top view and cross-sectional morphologies for CZTSSe absorbers for both $\mathbf{a}$ reference and $\mathbf{b}$ doped samples

Fig. 3 EDS line scans across the film thickness in a Na doping sample and $\mathbf{b}$ reference sample, respectively. The red regions show the positions of EDS line scans. The black dashed lines indicate the different layers in the structure while the red dashed line indicates the point at which the elemental compositions of the absorbers were obtained (a)
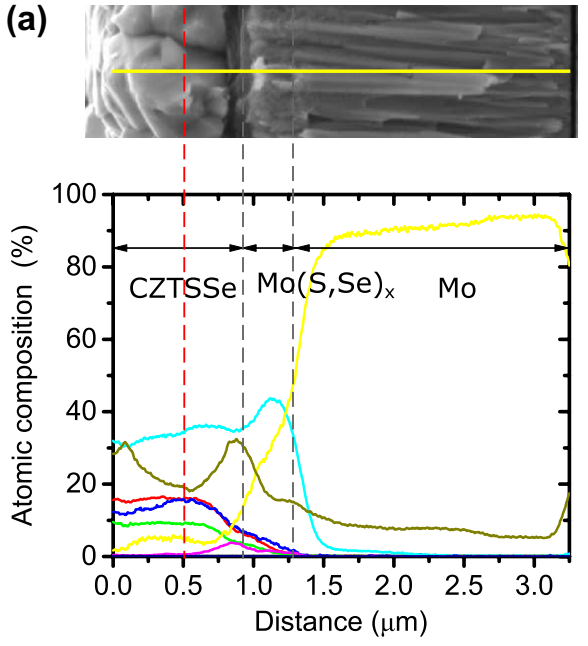

$\begin{array}{ll}-\mathrm{Cu} & -\mathrm{Zn} \\ \mathrm{S} & \mathrm{Mo}\end{array}$ (b)
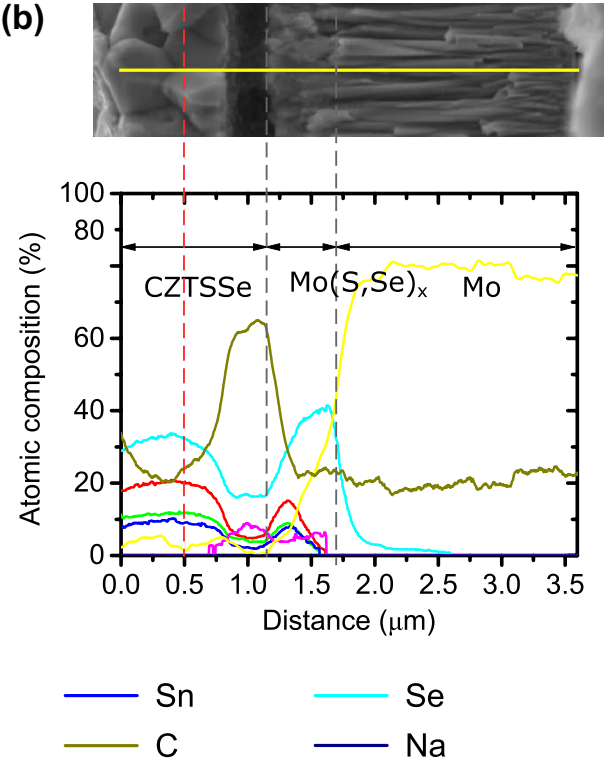

Table 2 Elemental compositions obtained from EDS line scans in the CZTSSe absorbers

\begin{tabular}{lllllll}
\hline & $\mathrm{Cu}$ & $\mathrm{Zn}$ & $\mathrm{Sn}$ & $\mathrm{Se}$ & $\mathrm{S}$ & $\mathrm{Se} /(\mathrm{S}+\mathrm{Se})$ \\
\hline Doped & 15.89 & 9.05 & 15.67 & 34.06 & 0.61 & 0.98 \\
Reference & 19.91 & 11.75 & 9.04 & 32.3 & 0.00 & 1 \\
\hline
\end{tabular}



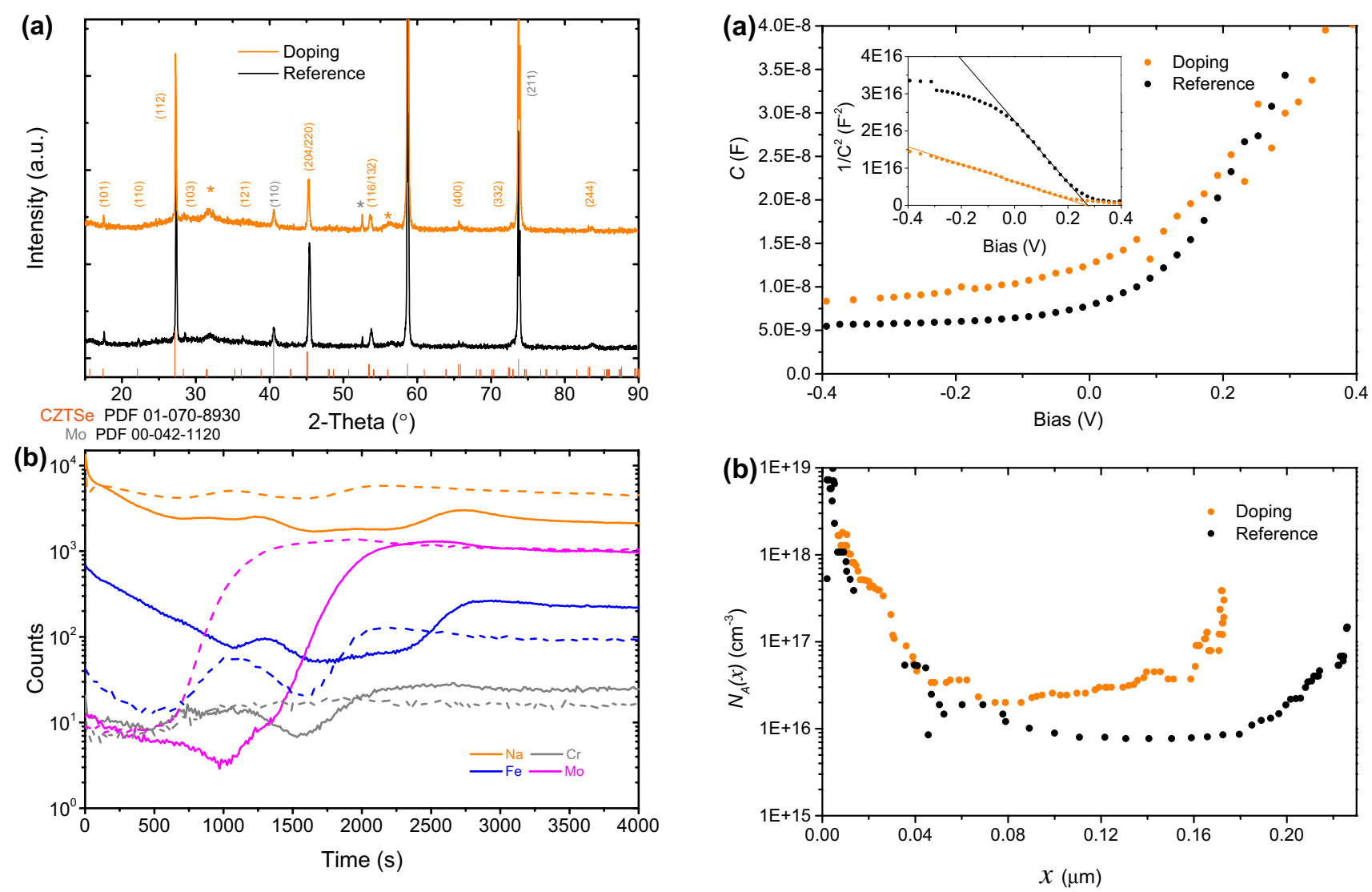

Fig. 4 a XRD pattern of CZTSSe absorber on Mo foil substrate prepared from doped solution and reference. b SIMS profile indicating the $\mathrm{Na}, \mathrm{Fe}, \mathrm{Cr}$, Mo content. The solid lines indicate the reference sample, and the dash lines indicate the doped sample

which is matched to the Mo pattern. For CZTSe patterns, the major peaks of (112), (204) and (312) planes appear at $27.34^{\circ}, 45.35^{\circ}$ and $53.7^{\circ}$, matching the polycrystalline kesterite crystal structure (PDF No: 01-070-8930) [28]. After doping, the texture coefficient $C_{h k l}$ increased to 1.02 for the (112) peak and (312) peak, but decreased to less than 1 for the (204). This indicates that the preferred orientation changes from (204) to (112) and (312) following the introduction of $\mathrm{Na}$. The peak at (110) also disappeared in the doped sample. The change in orientation preference can be related to a more uniform concentration of $\mathrm{Na}$ from the Mo interface upward during the selenisation. The calculated degree of preferred orientation $\sigma$ are 0.90 and 0.92 for doped and reference samples, respectively which indicates little change in crystallographic orientation between the samples [29].

SIMS measurements for $\mathrm{Na}$ and possible impurities are shown in Fig. 4b. To enable comparison between the doped and reference samples, the data were normalised to the same Mo counts level. The different rise times of Mo signal between two samples results from the different absorber thicknesses. The data clearly show that with

Fig. 5 a Capacitance-voltage characteristics (CV) of doped and reference devices. Inset: $1 / C^{2}$ versus potential profiles to determine builtin voltage. $\mathbf{b}$ The carrier concentration profile vs the distance from the junction interface

the exception of a small region towards the surface of the absorber, the $\mathrm{Na}$ level in the doped sample is higher than the reference sample throughout. Additionally, the amount of $\mathrm{Na}$ is also reasonably constant throughout the absorber demonstrating an intrinsic advantage of the solution doping approach over introducing additional an sodium-containing layer above or below the absorber. The source of background $\mathrm{Na}$ in the reference sample is likely to come from residual $\mathrm{Na}$ in the selinisation furnace [30]. Other impurities such as $\mathrm{Fe}$ and $\mathrm{Cr}$ were also present in both samples originating from the foil substrate although interestingly the doped sample also shows lower levels of $\mathrm{Cr}$ and Fe diffusing from foil substrate. It is possible that this is the origin of fewer grain boundaries observed in the Nadoped sample however, this requires further investigation to be conclusive.

In order to quantify the difference in Na concentration observed in the SIMS data, capacitance-voltage measurements $(C V)$ were performed with both forward and reverse AC biased voltage. These data are shown Fig. 5a for both the doped and reference devices. The relationship between 
bias voltage and capacitor is given by Mott Schottky equation [31],

$C(V)^{-2}=\frac{2\left(V_{b i}-V\right)}{q \varepsilon \varepsilon_{0} A^{2} N_{A}}$.

where $\mathrm{q}$ is the electron charge; $\varepsilon_{0}$ is the vacuum permittivity, $\varepsilon_{0}=8.85 \times 10^{-12} \mathrm{~F} \cdot \mathrm{m}^{-1} ; \varepsilon$ is the dielectric constant of kesterite, $\varepsilon=8.6$ is taken in this work based on the calculation from [32]; $A$ is the cell area. $V_{b i}$ is the built-in voltage and $N_{A}$ is the doping concentration. The Mott Schottky plot of $1 / C^{2}$ versus applied bias voltage is shown in the inset to Fig. 5a based on Eq. (2). The value of $V_{b i}$ for each device is estimated by the intersection of linear fits with voltage axis to be $271.6 \mathrm{mV}$ for the Na-doped sample and $273.5 \mathrm{mV}$ for the reference.

Within the depletion regions of studied films, there can be an additional dynamic response to the applied AC bias at the positions where the electronic levels of the defect cross the quasi-Fermi level. Therefore, the junction capacitance response can be expressed as a function of the distance from the junction interface $x$ which is related to the capacitance through,

$C=\frac{\varepsilon \varepsilon_{0} A}{x}$

The depletion width $\left(W_{d}\right)$ can be determined by using $\varepsilon \varepsilon_{0} A / C$ at zero bias[33]. It is found that doping changed $W_{d}$ from $0.16 \mu \mathrm{m}$ for reference to $0.10 \mu \mathrm{m}$. Further more, $N_{A}$ can be extracted by deriving Eq. (2) as follow:

$N_{A}=\frac{-2}{q \varepsilon \varepsilon_{0} A^{2}\left[d\left(1 / C^{2}\right) / d V\right]}$.

Based on the Eqs. (3 and 4), a plot of doping concentration and distance to the junction is drawn in Fig. 5b. As can be seen, the doping concentration is higher for the doped sample $\left(2.7 \times 10^{16} \mathrm{~cm}^{-3}\right)$ than reference $\left(7.7 \times 10^{15} \mathrm{~cm}^{-3}\right)$. These results support the observed improvement in the EQE for the Na-doped sample and are consistent with previous work in which higher carrier density in kesterite devices correlated with smaller depletion region widths [34].

\section{Conclusions}

In this work, a simple and novel approach to introducing Na-doping in flexible CZTS nanoparticle ink solar cells built on Mo-foil substrates is described. The introduction of Nadoping was found to improve the device performance from 2.5 to $4.4 \%$ and this was attributed to the formation of a more uniform thin film absorber morphology resulting in improved electron-hole pair generation. Further work will focus on optimising the doping concentration however the emphasis here is that the doping approach lends itself to high volume manufacturing as a result of solution processing. An additional key benefit is that the doping profile is reasonably constant throughout the device. This is in contrast to doping techniques which rely in the introduction of an additional thin film layer into the device structure.

Acknowledgements This work was supported by the EPSRC North East Centre for Energy Materials (EP/R021503/1) and also EPSRC Grant (EP/N024389/1).

Open Access This article is distributed under the terms of the Creative Commons Attribution 4.0 International License (http://creativeco mmons.org/licenses/by/4.0/), which permits unrestricted use, distribution, and reproduction in any medium, provided you give appropriate credit to the original author(s) and the source, provide a link to the Creative Commons license, and indicate if changes were made.

\section{References}

1. A. Chirilă, P. Reinhard, F. Pianezzi, P. Bloesch, A.R. Uhl, C. Fella, L. Kranz, D. Keller, C. Gretener, H. Hagendorfer et al., Nat. Mater. 12(12), 1107 (2013)

2. S. Lopez-Marino, Y. Sanchez, M. Espindola-Rodriguez, X. Alcobe, H. Xie, M. Neuschitzer, I. Becerril, S. Giraldo, M. Dimitrievska, M. Placidi, L. Fourdrinier, V. Izquierdo-Roca, A. PerezRodriguez, E. Saucedo, J. Mater. Chem. A 4, 1895 (2016). https ://doi.org/10.1039/C5TA09640E

3. C.M. Sutter-Fella, J.A. Stückelberger, H. Hagendorfer, F. La Mattina, L. Kranz, S. Nishiwaki, A.R. Uhl, Y.E. Romanyuk, A.N. Tiwari, Chem. Mater. 26(3), 1420 (2014)

4. W.H. Oo, J. Johnson, A. Bhatia, E. Lund, M. Nowell, M.A. Scarpulla, J. Electron. Mater. 40(11), 2214 (2011)

5. T. Prabhakar, N. Jampana, Sol. Energy Mater. Sol. Cells 95(3), $1001(2011)$

6. P. Salomé, V. Fjällström, A. Hultqvist, M. Edoff, IEEE J. Photovolt. 3(1), 509 (2013)

7. J.V. Li, D. Kuciauskas, M.R. Young, I.L. Repins, Appl. Phys. Lett. 102(16), 163905 (2013)

8. G. Altamura, C. Roger, L. Grenet, J. Bleuse, H. Fournier, S. Perraud, H. Mariette, MRS Online Proc. Libr. Arch. 1538, 103 (2013)

9. A. Collord, H. Xin, H. Hillhouse, IEEE J. Photovolt. 5(1), 288 (2015)

10. R.H. Doremus, J. Phys. Chem. 68(8), 2212 (1964)

11. S. López-Marino, Y. Sánchez, M. Espíndola-Rodríguez, X. Alcobé, H. Xie, M. Neuschitzer, I. Becerril, S. Giraldo, M. Dimitrievska, M. Placidi et al., J. Mater. Chem. A 4(5), 1895 (2016)

12. P. Salomé, H. Rodriguez-Alvarez, S. Sadewasser, Sol. Energy Mater. Sol. Cells 143, 9 (2015)

13. I. Becerril-Romero, L. Acebo, F. Oliva, V. Izquierdo-Roca, S. López-Marino, M. Espíndola-Rodríguez, M. Neuschitzer, Y. Sánchez, M. Placidi, A. Pérez-Rodríguez et al., Prog. Photovolt. 26(1), 55 (2018)

14. E. Ghorbani, J. Kiss, H. Mirhosseini, M. Schmidt, J. Windeln, T.D. Kühne, C. Felser, J. Phys. Chem. C 120(4), 2064 (2016)

15. W. Wang, M.T. Winkler, O. Gunawan, T. Gokmen, T.K. Todorov, Y. Zhu, D.B. Mitzi, Adv. Energy Mater. 4(7), 1301465 (2014)

16. C.J. Hages, M.J. Koeper, C.K. Miskin, K.W. Brew, R. Agrawal, Chem. Mater. 28(21), 7703 (2016)

17. S.G. Haass, C. Andres, R. Figi, C. Schreiner, M. Bürki, Y.E. Romanyuk, A.N. Tiwari, Adv. Energy Mater. 8(4), 1701760 (2018) 
18. S.G. Haass, Alkali treatment of solution processed kesterite solar cells. Ph.D. thesis, ETH Zurich (2017). https://doi.org/10.3929/ ethz-b-000235925

19. G. Altamura, M. Wang, K.L. Choy, Sci. Rep. 6, 22109 (2016)

20. P.R. Ghediya, T.K. Chaudhuri, J. Mater. Sci.: Mater. Electron. 26(3), 1908 (2015)

21. Z. Zhou, Y. Wang, D. Xu, Y. Zhang, Sol. Energy Mater. Sol. Cells 94(12), 2042 (2010)

22. X. Xu, Y. Qu, V. Barrioz, G. Zoppi, N.S. Beattie, RSC Adv. 8, 3470 (2018). https://doi.org/10.1039/C7RA13336G

23. Y. Qu, G. Zoppi, N.S. Beattie, Prog. Photovolt. 24(6), 836 (2016)

24. Y.S. Lee, T. Gershon, O. Gunawan, T.K. Todorov, T. Gokmen, Y. Virgus, S. Guha, Adv. Energy Mater. 5(7), 1401372 (2015)

25. A. Nakane, H. Tampo, M. Tamakoshi, S. Fujimoto, K.M. Kim, S. Kim, H. Shibata, S. Niki, H. Fujiwara, J. Appl. Phys. 120(6), 064505 (2016)

26. N. renewable energy laboratory. Reference solar spectral irradiance: Air mass 1.5. https://rredc.nrel.gov/solar/spectra/am1.5/
27. C.K. Miskin, W.C. Yang, C.J. Hages, N.J. Carter, C.S. Joglekar, E.A. Stach, R. Agrawal, Prog. Photovolt. 23(5), 654 (2015)

28. I. Olekseyuk, L. Gulay, I. Dydchak, L. Piskach, O. Parasyuk, O. Marchuk, J. Alloys Compds. 340(1), 141 (2002). https://doi. org/10.1016/S0925-8388(02)00006-3

29. G. Zoppi, K. Durose, S. Irvine, V. Barrioz, Semicond. Sci. Technol. 21(6), 763 (2006)

30. D. Colombara, U. Berner, A. Ciccioli, J.C. Malaquias, T. Bertram, A. Crossay, M. Schöneich, H.J. Meadows, D. Regesch, S. Delsante et al., Sci. Rep. 7, 43266 (2017)

31. S.K. Saha, A. Guchhait, A.J. Pal, Phys. Chem. Chem. Phys. 14(22), 8090 (2012)

32. C. Persson, J. Appl. Phys. 107(5), 053710 (2010)

33. A. Guchhait, Z. Su, Y.F. Tay, S. Shukla, W. Li, S.W. Leow, J.M.R. Tan, S. Lie, O. Gunawan, L.H. Wong, ACS Energy Lett. 1(6), 1256 (2016)

34. H.S. Duan, W. Yang, B. Bob, C.J. Hsu, B. Lei, Y. Yang, Adv. Funct. Mater. 23(11), 1466 (2013) 